\title{
Modèles d'imprégnation musicale en Afghanistan
}

John Baily et Veronica Doubleday

\section{(2) OpenEdition \\ Journals}

Édition électronique

URL : http://journals.openedition.org/ethnomusicologie/2301

ISSN : 2235-7688

Éditeur

ADEM - Ateliers d'ethnomusicologie

Édition imprimée

Date de publication : 1 janvier 1988

Pagination : 112-124

ISBN : 2-8257-0159-9

ISSN : $1662-372 X$

Référence électronique

John Baily et Veronica Doubleday, « Modèles d'imprégnation musicale en Afghanistan », Cahiers d'ethnomusicologie [En ligne], 1 | 1988, mis en ligne le 15 août 2011, consulté le 19 avril 2019. URL : http://journals.openedition.org/ethnomusicologie/2301 


\title{
MODÈLES D'IMPRÉGNATION MUSICALE EN AFGHANISTAN*
}

\author{
John Baily et Veronica Doubleday
}

L'étude de l'imprégnation musicale est une clef pour mieux comprendre les systèmes musicaux en tant que phénomènes cognitifs, car elle montre comment se construisent des schèmes cognitifs et quelles sont les informations nécessaires à ce processus. Elle dévoile ainsi la nature du savoir musical, tout en permettant de différencier ses éléments innés de ceux acquis socialement. Partant de l'environnement musical de l'enfant, notre analyse se propose d'en examiner l'influence sur le développement de la pratique musicale. La société afghane étant fermement ancrée dans la vie domestique et familiale, les compétences musicales de l'enfant sont déterminées par celles des adultes qui l'entourent de près. C'est ainsi que nous envisageons le développement musical comme un processus social intimement lié au rôle que l'enfant sera appelé à jouer, en tant que musicien, dans sa vie d'adulte.

Les données sur lesquelles se fonde notre étude furent recueillies dans la ville de Hérat et ses environs au cours des années 1970. Hérat est tout à fait représentative d'une ville de province de l'Afghanistan. Quant à Kaboul, la capitale, les conditions de vie y sont à maints égards assez différentes. Depuis notre enquête, l'Afghanistan a été envahi par l'Union soviétique, et Hérat troisième ville du pays - a été dévastée par la guerre. Nombre de ses habitants ont été tués ou se sont réfugiés en Iran, au Pakistan et dans d'autres pays. Si le cadre général de notre analyse s'applique sans doute également aux conditions prévalant à l'heure actuelle, nous avons néanmoins préféré employer le passé pour rappeler que toutes les sphères de la vie afghane ont été considérablement bouleversées, y compris et peut-être spécialement celle de la musique.

\section{La signification de la musique à Hérat}

Il importe d'évoquer tout d'abord certains aspects du milieu socio-culturel dans lequel se déroulait l'apprentissage musical, dans la mesure notamment où ils étaient influencés par les croyances et les pratiques liées à l'Islam.

La définition afghane de la "musique" ne coïncide pas totalement avec celle qui a cours en Europe. Ce que nous désignons ici par ce terme n'est pas nécessairement ce que les Afghans qualifieraient de la sorte. Du point de vue afghan, la musique (musiqi) impliquait le jeu d'un instrument, en particulier d'un instrument mélodique. Par contre, le chant non accompagné n'entrait 
pas dans la catégorie de musiqi, qu'il s'agisse du chant religieux (notamment l'appel à la prière par le muezzin) ou encore du chant des femmes, même lorsque celui-ci était accompagné du tambour-sur-cadre (däireh). Pour les fins de l'analyse, nous avons néanmoins inclu ces manifestations dans notre propos $^{1}$.

Les gens de Hérat formaient une société musulmane traditionnelle où le purdah, c'est-à-dire la séparation des sexes, était strictement observée. Les hommes dominaient l'espace public, alors que les femmes étaient réléguées dans l'intimité de la sphère domestique. A l'extérieur du cadre familial, les rencontres sociales étaient également régies par ce principe, de même que la pratique musicale. En dehors du théâtre avec ses courtisanes, il était rare de voir garçons et filles ou hommes et femmes faire de la musique ensemble. Lors des noces - occasion de prédilection pour s'adonner à la danse, au chant ou à la musique instrumentale - , hommes et femmes occupaient des espaces distincts, souvent des maisons continguës. Il existait des différences notables dans les styles musicaux, les instruments, les répertoires et les connaissances musicales des hommes et des femmes. Ainsi le purdah avait-il une influence sur la pratique de la musique.

Dans le système de valeurs de Hérat, la signification de la musique était plutôt ambiguë. Si les habitants étaient généralement d'accord pour dire qu'elle jouait un rôle dans les rites et les cérémonies, ils n'en invoquaient pas moins l'autorité religieuse pour la rejeter quand elle répondait à d'autres fins, tel le divertissement. En effet, les mullahs orthodoxes (sunnites haniffites) proclament que la musiqi - c'est-à-dire la musique instrumentale - est un mal, voire même un péché. Cette vision austère, pour ne pas dire puritaine, est toutefois contrebalancée par les traditions populaires et soufiques qui valorisent l'apport spirituel de la musique, en décrivant cette dernière comme une "nourriture de l'âme". Dans certains contextes, la musique est même utilisée à des fins spirituelles.

On distinguait clairement entre musiciens amateurs et musiciens professionnels (Baily 1979). Il revenait aux familles héréditaires de musiciens professionnels, appelés sāzandeh, d'animer les réunions publiques ou semipubliques, tels les noces et les concerts. Les hommes säzandeh des villes chantaient et jouaient de l'armonia, petit harmonium à soufflet manuel largement répandu en Asie du Sud, de la paire de tambours tabla, ainsi que du luth rubāb et d'autres cordophones (delrubā, tānpurā et sormandel). Les sāzandeh avaient un statut ambivalent: d'un côté, c'était des artistes (honarmand) qu'il fallait admirer à l'instar des poètes, des peintres et des calligraphes et qui avaient ainsi acquis un peu du prestige détenu jadis par les musiciens de la cour de Kaboul; de l'autre côté, on les qualifiait de jat, leur imputant toutes sortes de pratiques contraires aux préceptes de l'Islam. Les femmes sāzandeh de Hérat chantaient et jouaient de l'harmonium, des tabla et du däireh (tambour-sur-cadre), mais non pas d'instruments à cordes, ceux-ci étant réservés aux hommes. Comme ces femmes exerçaient un métier qui les amenait à enfreindre continuellement les règles du purdah, elles occupaient un statut social nettement inférieur, tout en jouissant d'une certaine notoriété ; souvent, on les traitait de "femmes faciles", quand on ne les qualifiait pas de 
prostituées. Vis-à-vis du public, les musiciens amateurs œuvraient pour se démarquer des säzandeh, en insistant sur le fait qu'ils jouaient et chantaient, eux, par amour pour la musique et non pas simplement pour gagner leur vie; ils revendiquaient le statut de shauqi (enthousiaste) - en dépit du fait que certains d'entre eux tiraient également leur subsistance de la musique ${ }^{2}$.

L'éducation musicale ne faisait pas partie du cursus scolaire, qu'il soit traditionnel ou moderne. L'école coranique offrait une éducation de base ; et il n'est guère surprenant que, vu l'attitude des mullahs, la musique n'y avait aucune place. Elle n'était pas non plus enseignée dans les écoles laïques modernes qui ne reconnaissaient pas plus la valeur éducative des chansons et des jeux didactiques. Il existait une théorie formalisée de la musique dite "science musicale", mais c'était là un domaine réservé aux hommes sāzandeh des villes et non pas un savoir accessible à tous. Elle comprenait le sargam, système indien de notation orale et écrite. Seuls les enfants mâles des familles héréditairement musiciennes avaient accès à ce savoir formel, ainsi qu'un nombre restreint d'enfants dont les parents avaient entrepris la démarche peu commune de leur offrir des leçons privées de musique.

\section{Quatre modèles d'imprégnation musicale}

Les enfants de Hérat ne possédaient pas une culture musicale distinctive en vertu de laquelle ils auraient créé leurs propres chansons. Il n'y avait pratiquement aucun répertoire enfantin, pas plus que des chansons d'adultes destinées aux enfants. En faisant de la musique, les enfants de Hérat imitaient avant tout leurs aînés: leurs chansons étaient le plus souvent celles que l'on entendait couramment à la radio, et leurs danses rappelaient celles que femmes et hommes exécutaient à l'occasion des cérémonies de mariage.

Les berceuses constituaient une expérience musicale primordiale commune à la quasi-totalité des enfants de Hérat. Les femmes les chantaient à leurs bébés emmaillotés et à leurs enfants en bas âge, en les berçant vigoureusement. Ce type de chant était fortement rythmé ; il s'agissait essentiellement de la répétition de la formule Huwa 'lläh («Il est Dieu»), mais parfois la mère interprétait une longue série de vers appropriés.

Passons maintenant au processus d'apprentissage de la musique, en considérant quatre catégories distinctes d'enfants: garçons et filles d'une part, enfants de familles ordinaires (non musiciennes) et de familles de musiciens héréditaires d'autre part. Il importe de signaler que le nombre de sāzandeh dans une ville comme Hérat est relativement bas: il y avait en tout une trentaine de femmes et une vingtaine d'hommes appartenant à cette catégorie.

\section{Les filles de familles non musiciennes}

Du point de vue de l'apprentissage et de la participation, les filles avaient accès à ce que l'on pourrait appeler la "musique domestique des femmes". Celle-ci comprenait le chant soit en soliste, soit en groupe, accompagné du seul instrument important des femmes, à savoir le dāireh dont le cadre était 


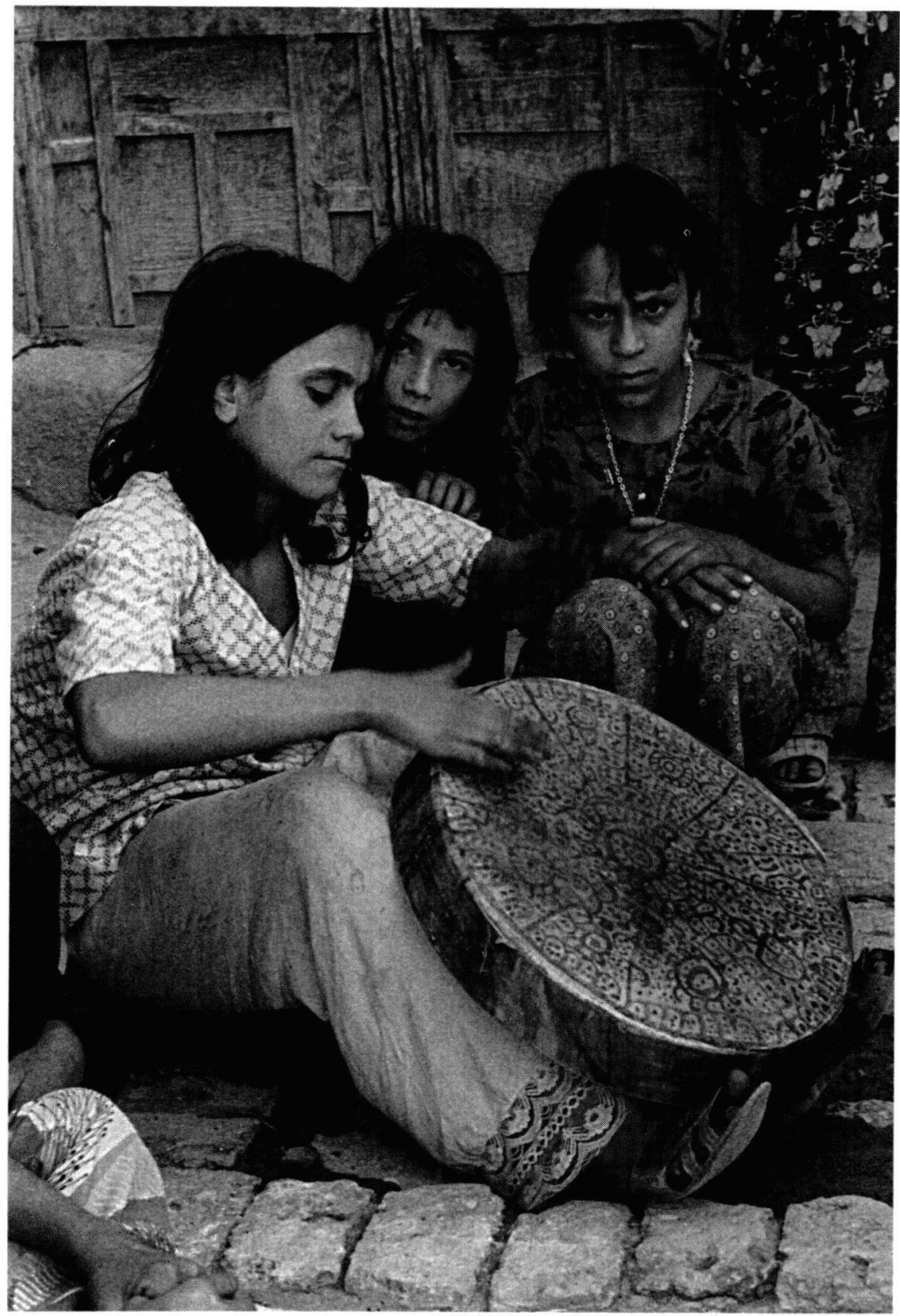

Filles de familles non musiciennes jouant du tambour-sur-cadre dāireh. Hérat, Afghanistan, 1975 (photo: Veronica Doubleday). 
muni d'anneaux et de petits grelots. Ce tambour était aussi utilisé pour rythmer certaines danses exécutées par les femmes pour célébrer un événement heureux, comme les fiançailles, le mariage ou encore la septième nuit après la naissance d'un enfant. D'une manière générale, les fillettes ne tardaient pas à se joindre aux musiciennes en battant des mains sur le rythme du däireh, notamment lorsqu'il s'agissait d'encourager une danseuse solo, ni à reprendre le refrain de la chanson. Certaines chansons étaient traditionnelles, d'autres d'origine populaire et transmises par Radio Afghanistan ou la radio iranienne. De telles occasions offraient des circonstances idéales permettant à la fille d'écouter et d'apprendre la musique, tout en y prenant une part active aux côtés d'interprètes adultes qualifiées et motivées.

Pour les enfants, en particulier les fillettes, ce type de pratique musicale équivalait à un jeu auquel on pouvait se livrer seul ou sous l'égide de filles plus âgées ou de femmes adultes. Si la famille ne possédait pas de dāireh, elle l'empruntait aux voisins. Les fillettes se contentaient souvent d'un plateau en métal; parfois, elles imitaient même les tabla des ensembles féminins en se servant d'une cuvette et d'un seau renversés. Le jeu du tambour et les battements des mains faisaient partie intégrante de ces séances de chant et de danse, et les rythmes étaient appris en bas âge. A peine capables de marcher, les fillettes étaient encouragées à danser : on les poussait parfois à faire étalage de leurs talents précoces devant des invités. Le jeu du dāireh exigeait sans doute une certaine habileté, mais les femmes, loin d'y voir un art qu'il fallait apprendre à dessein, considéraient cela comme allant de soi. Déjà toutes petites, les filles entendaient les différents rythmes du däireh et apprenaient à les reproduire en regardant faire leurs aînées.

Avant d'atteindre la puberté, les filles disposaient de beaucoup de temps pour faire la musique; souvent, elles finissaient par se passionner pour ce divertissement. Mais quand elles approchaient la puberté, la pratique de la musique était découragée, car il importait alors de faire montre de sens des responsabilités, de modestie et d'assiduité au travail domestique. Sans que leurs talents musicaux ne fussent oubliés, elles avaient alors moins d'occasions de les appliquer, et tout désir de faire de la musique devait être réfréné: les jeunes filles savaient bien qu'elles allaient bientôt se marier et que les femmes plus âgées à la recherche d'une belle-fille avaient commencé à scruter leurs manières et leurs talents de ménagères. Un intérêt marqué envers la musique risquait d'être interprété comme un signe de frivolité et de répugnance aux travaux domestiques.

\section{Les garçons de familles non musiciennes}

Les garçons baignaient, eux aussi, dans la musique domestique des femmes. Ils y participaient même jusqu'à un certain point : ils chantaient et se mêlaient aux danses, mais ils fuyaient le dāireh qu'ils considéraient comme l'apanage des filles. Les villages connaissaient une tradition de chant solo pendant les travaux des champs. Les soirs du mois du Ramadan (jeûne musulman) étaient pour les garçons l'occasion de chanter en groupe : ils allaient de maison en maison pour interpréter des couplets de circonstance et faire la quête. 


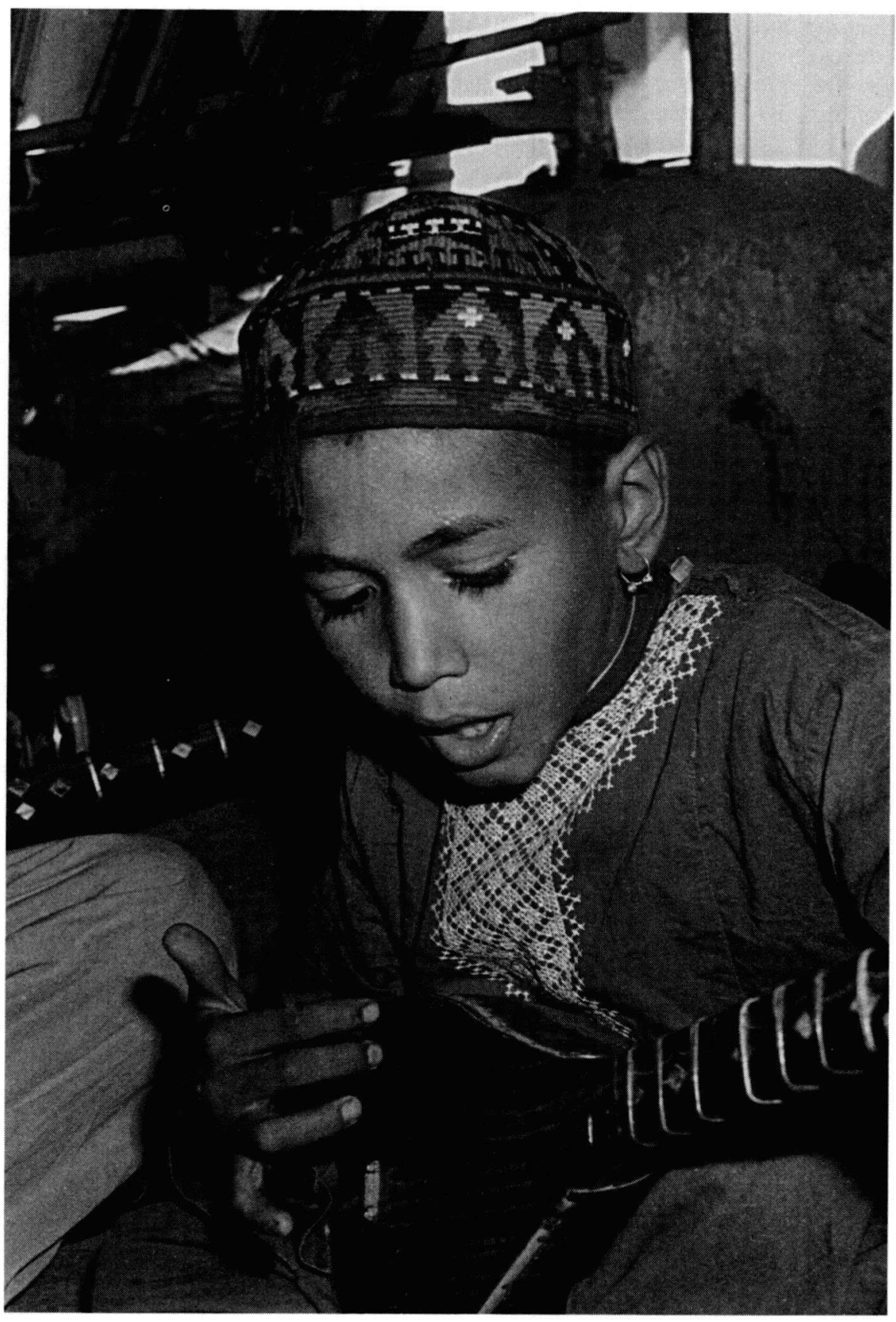

Garçon d'une famille non musicienne chantant et jouant du dutār. Hérat, Afghanistan, 1974 (photo: John Baily). 
Quelques garçons confectionnaient leurs propres instruments en utilisant une cuiller en bois pour fabriquer un luth à long manche (dutār), ou des boîtes de conserves pour obtenir des tambours. Peu de garçons avaient accès aux instruments de musique des adultes, qui n'étaient d'ailleurs pas des objets courants dans les ménages de Hérat. Les instruments étaient onéreux, délicats et dans l'ensemble plutôt mal vus, si bien qu'on les gardait bien à l'abri des mains d'enfant. Peut-être le dutār était-il l'instrument le plus couramment pratiqué par les amateurs de Hérat, tant en solo qu'en accompagnement. Une enquête sur la manière dont les joueurs adultes de dutär s'étaient formés (Baily 1976) a révélé que la plupart d'entre eux prétendaient être autodidactes et en étaient fiers. Le plus souvent, ils commençaient à jouer du dutār vers l'âge de douze ans, en imitant un autre interprète, généralement un parent ou un ami, mais sans révéler ce qu'ils étaient en train de faire. Ils allaient écouter et observer, puis pratiquer en cachette. Pour avoir accès à un instrument, ils devaient souvent l'emprunter à la dérobée lorsque son propriétaire était loin de la maison. L'acquisition d'un dutār, le plus souvent un instrument d'occasion acheté au luthier, comptait énormément pour l'aspirant musicien. Souvent, les parents décourageaient leurs fils de pratiquer la musique, leur disant que c'était un péché, si bien que l'apprentissage du dutār était jonché de nombreux obstacles.

Or les garçons jouissaient de plus de liberté que les filles pour élargir leurs connaissances musicales au fur et à mesure qu'ils grandissaient. Ils étaient notamment en mesure de se réunir en groupe d'amis pour faire la musique, bénéficiant ainsi d'une possibilité supplémentaire d'apprendre par participation. Quelques musiciens amateurs prenaient des leçons payantes, généralement auprès d'un musicien professionnel, afin d'apprendre certaines compositions et d'approfondir leurs connaissances en matière de théorie musicale.

\section{Les filles de familles de musiciens héréditaires}

Dans certaines familles de musiciens héréditaires, tant les femmes que les hommes étaient des professionnels, alors que dans d'autres, seuls les hommes exerçaient ce métier, les femmes étant soumises au purdah le plus strict. Les enfants des familles dont les femmes étaient également musiciennes de métier grandissaient dans un environnement familial entièrement consacré à la pratique musicale. L'ensemble féminin, qui animait les noces, comptait généralement quatre ou cinq membres et interprétait des chansons populaires de la radio, ainsi que des chants traditionnel de Hérat et de la musique de danse, en utilisant l'harmonium, les tabla et un ou plusieurs dāireh. Comme les cérémonies de mariage duraient vingt-quatre heures, les musiciennes s'y rendaient avec leurs bébés et leurs enfants en bas âge - jour après jour pendant la saison des fêtes, moins fréquemment à d'autres moments de l'année. Dès le début, leurs enfants baignaient dans la musique. Les fillettes faisaient des courses et s'occupaient de leurs cadets, et il était admis qu'elles apprenaient beaucoup en regardant et en écoutant leurs mères, leur tantes, leurs sœurs et leurs cousines. 


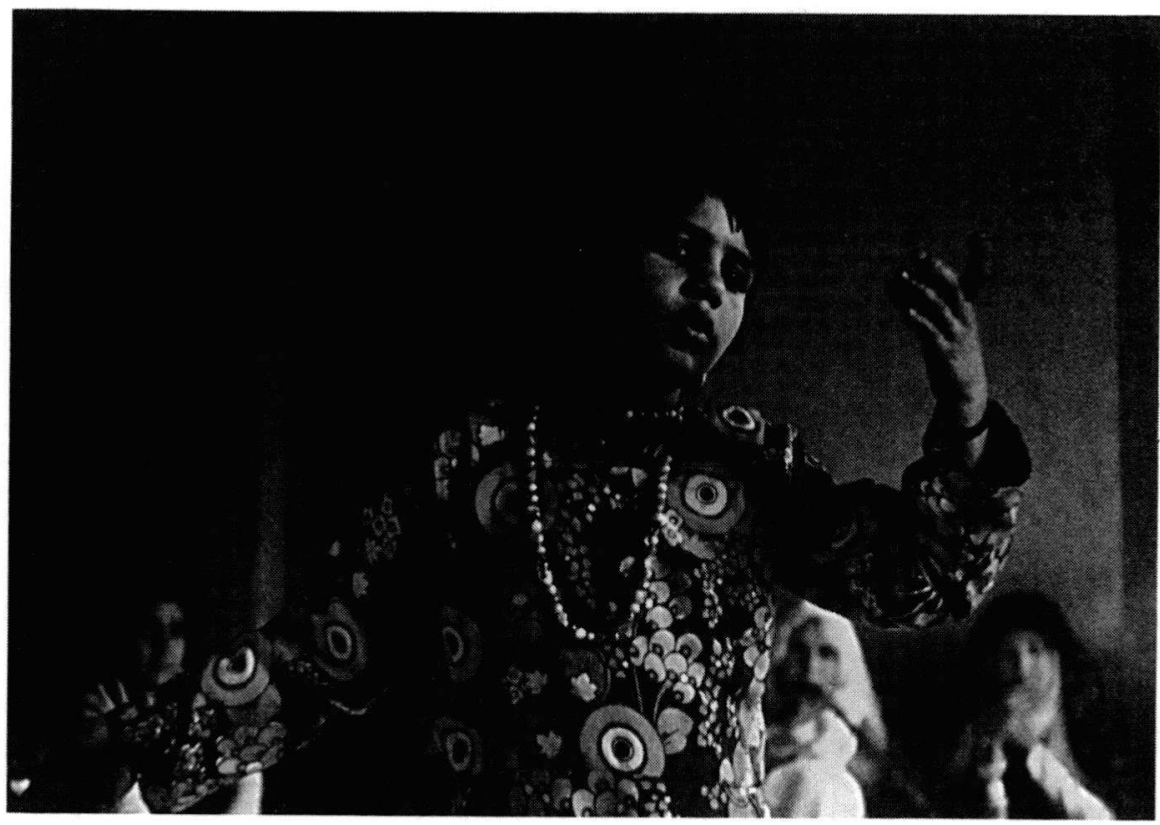

Fille d'une famille de musiciens dansant pour divertir les femmes lors d'une fête de mariage. Hérat, Afghanistan, 1977 (photo: Veronica Doubleday).

Vers l'âge de dix ans, les filles commençaient à chanter et à danser en soliste lors des noces, à la demande de leurs hôtes qui les récompensaient ensuite par une petite somme d'argent. Contrairement aux filles des familles non musiciennes, elles n'étaient ni découragées de pratiquer la musique ni exhortées à se comporter avec modestie ; plutôt, elles cherchaient à se faire remarquer, et on les récompensait quand elles faisaient preuve d'extroversion et de confiance en soi. Peu après, une telle fille était encouragée à jouer du däireh et à chanter au sein d'un ensemble. Ce dernier était organisé d'une manière souple, permettant à chacune des interprètes de se retirer temporairement pour se reposer ou nourrir son enfant. Ses membres se recrutaient dans le groupe de parenté ou d'alliance, et toutes les filles choisies devenaient des chanteuses et des joueuses de dãireh compétentes.

Ensuite la jeune musicienne aspirait à maîtriser les tabla. Le jeu de cette paire de tambours était une adaptation de la technique du dāireh, et les frappes à main plate qu'il exigeait, différaient sensiblement de celles employées par les hommes qui suivaient le style classique indien. Les tambours des femmes (qui étaient importés du Pakistan) étaient de qualité nettement inférieure, et les femmes renonçaient même à les accorder. Certaines filles n'acquéraient jamais une compétence suffisante pour jouer des tabla et occupaient de ce fait un rôle subalterne en tant que chanteuses et 
joueuses de dãireh. Grâce à leur compétence aux tabla, un petit nombre de jeunes filles accédaient ensuite à l'harmonium, en apprenant seules et en reproduisant des mélodies familières. Au début, ces filles jouaient surtout des airs de danse; puis elles se mettaient à combiner le rôle de chanteuse principale avec le jeu de l'harmonium. Vers l'âge de quatorze ans environ, une jeune fille douée était ainsi en mesure de diriger l'ensemble, de choisir son répertoire et d'élaborer un programme musical d'une demi-heure; à l'âge de seize ou dix-sept ans, elle était devenue une musicienne accomplie, capable d'assumer la direction d'un ensemble.

Contrairement aux musiciens professionnels des villes, les musiciennes n'étaient pas poussées à affiner leur technique de jeu; elles ne bénéficiaient pas de cours, mise à part la correction d'erreurs. Dans le cadre des ensembles, les femmes encourageaient et louaient les apprenties, mais en dernier ressort chaque fille devait ressentir sa propre motivation et faire son possible pour apprendre par imitation. Si toutes les filles étaient censées savoir chanter et jouer du däireh, la maîtrise des tabla et de l'harmonium exigeait un apprentissage, même si celui-ci se faisait en autodidacte ${ }^{3}$.

\section{Les garçons de familles de musiciens héréditaires}

Les garçons élevés dans les familles de musiciens professionnels grandissaient dans un monde musical plus complexe. En plus du répertoire populaire exécuté aussi par les femmes, les hommes säzandeh des villes interprétaient différents genres de musique savante (étroitement liés à la musique «classique» de l'Inde et du Pakistan) inconnus des femmes. Leur prérogrative était la théorie musicale, dans l'ensemble une variante de celle de la musique hindoustanie avec ses räga et tâla. Les musiciens professionnels insistaient sur la nécessité d'apprendre la musique par l'intermédiaire de la notation, ainsi que sur l'importance de la relation privilégiée de maître à disciple dans la transmission du savoir musical. Mais en dépit de ces idéaux, il semble que les garçons sāzandeh apprenaient la musique de la même manière que les filles de cette catégorie, c'est-à-dire "d'oreille» en étant fréquemment confrontés à la musique, et par tâtonnements individuels dans un environnement familial où la pratique musicale était fortement encouragée. Il n'y avait pas d'apprentissage formel, ni d'exercices du type de ceux employés pour apprendre la musique hindoustanie, ni de véritables "séances de pratique".

Les sāzandeh avaient des idées intéressantes au sujet du savoir musical et de son développement. Ils admettaient que certains individus étaient naturellement doués pour la musique et pensaient qu'il fallait vivifier ce don, comme on souffle sur les braises pour qu'elles s'enflamment. Ils croyaient que l'acquisition de compétences musicales dépendait étroitement du fait de baigner très jeune dans la musique, et ils avaient coutume d'emmener leurs fils en bas âge s'asseoir avec eux sur le podium lorsqu'ils allaient jouer en public. Ils croyaient que même en dormant, l'enfant continuait à absorber les sons. Souvent, un garçon manifestait un vif intérêt envers un instrument particulier quand il était encore très jeune (cinq ou six ans), et on l'autorisait alors à s'y essayer. Les parents intervenaient bien évidemment dans ce processus, 


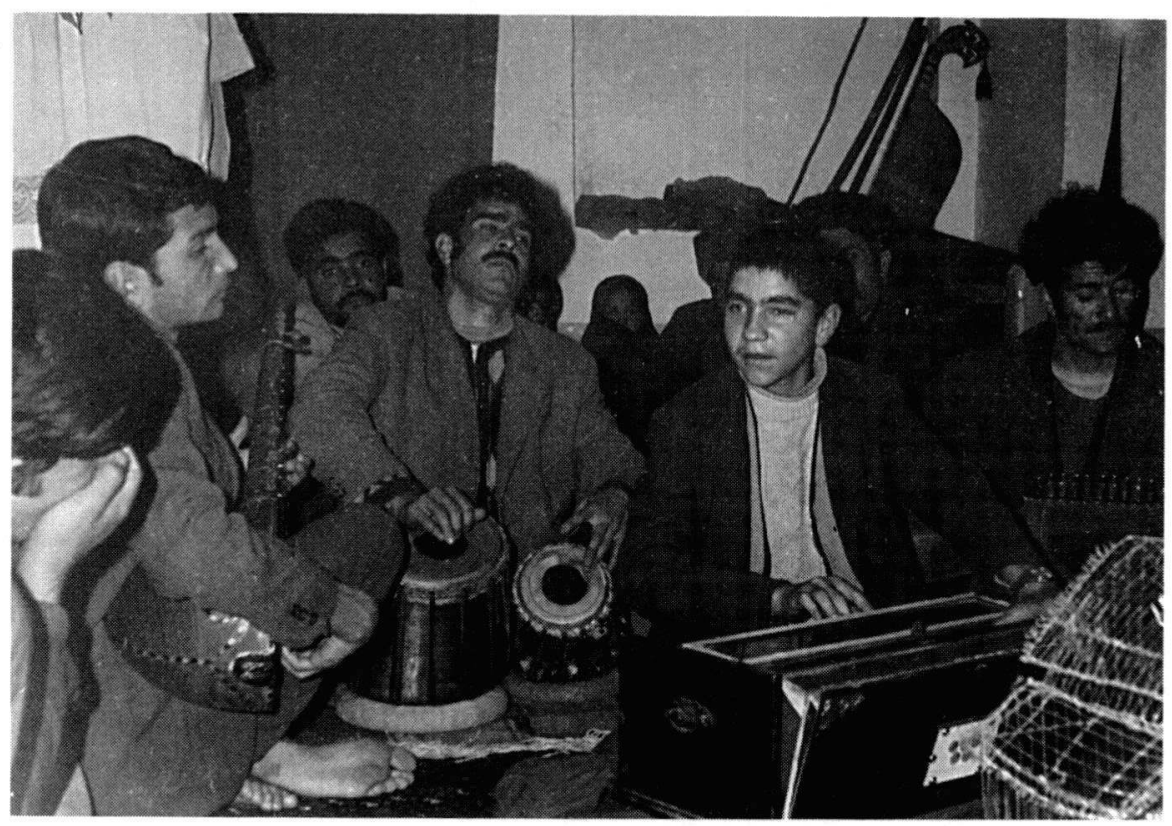

Jeune chanteur et joueur d'harmonium de treize ans d'une famille de musiciens, dirigeant l'orchestre. A ses côtés, de droite à gauche: deux de ses frères, jouant du rubāb et des tabla, et un joueur de dutār venant d'une famille non musicienne. Hérat, Afghanistan, 1974 (photo: John Baily).

davantage en encourageant le garçon quand il jouait juste qu'en le décourageant lorsqu'il jouait faux. Il semble cependant qu'en dépit de tout ce discours sur la manière idéale d'apprendre la musique, les garçons des familles de musiciens apprenaient en fait par imitation. En étant encore petits, et s'ils venaient d'une famille dont les femmes étaient, elles aussi, musiciennes, ils bénéficiaient en outre - comme les filles - de la possibilité d'accompagner leur mère aux cérémonies de mariage, voire même de participer aux prestations musicales, en particulier par le jeu des tabla; mais cette phase se terminait quand ils atteignaient la puberté.

Vers l'âge de douze ans, quand les jeunes musiciens amateurs commençaient à apprendre un instrument, le jeune sāzandeh était prêt à se lancer dans une carrière professionnelle en s'insérant dans un ensemble. Une fois devenu un membre régulier et actif du groupe, il avait la possibilité de progresser davantage par le simple fait de jouer avec d'autres. Un jeune chanteur et joueur d'harmonium âgé de douze ans était à même de diriger un ensemble pour une série de chansons; quand il avait seize ans, il était susceptible de diriger l'ensemble régulièrement. Un musicien se faisait connaître comme joueur d'un instrument particulier, mais il était dans bien des cas capable d'en jouer d'autres, quoique le plus souvent en privé plutôt qu'en public - ce qui était vu comme un signe de maturité musicale. 
Dans ces familles, on rencontrait quelques non musiciens. Ceux-ci n'étaient pas nécessairement dépourvus de talent, mais ils avaient opté pour un autre métier, quand on ne les avait pas encouragé à le faire. Les ensembles de säzandeh étaient le plus souvent des entreprises de famille, n'offrant du travail à temps complet qu'à un nombre restreint de musiciens professionnels. Les garçons tendaient à apprendre les instruments dont l'ensemble familial allait avoir besoin dans les années à venir ${ }^{4}$.

\section{Commentaire}

L'aspect le plus intéressant des données réunies ici réside probablement dans le contraste existant entre les enfants des familles de musiciens professionnels et ceux des familles non musiciennes: on s'aperçoit que les premiers possédaient davantage de compétences musicales en termes de capacités motrices et cognitives, ainsi qu'un répertoire plus riche. Or cet écart ne relevait pas d'une différence entre ce que l'on pourrait appeler des modes formels et informels d'apprentissage de la musique. Il serait d'ailleurs abusif de parler dans ce contexte d'éducation ou de pratique, car ces termes sous-entendent «un processus d'apprentissage direct " (Merriam 1964 : 146) ainsi qu'une situation d'apprentissage délibérément établie, destinée à la transmission d'informations. Tout cela n'était guère le cas à Hérat. Les enfants des familles de musiciens tout comme ceux des familles ordinaires apprenaient de la même manière, par imitation et participation dans un contexte pouvant être qualifié d'autoinstruction suivant un rythme déterminé individuellement. Les variations dans les compétences musicales propre aux deux groupes d'enfants résultaient ainsi d'environnements sociaux différents: les situations et les processus d'apprentissage disponibles étaient inhérents au mode de vie qui distinguait les familles ordinaires de celles de musiciens professionnels.

L'imitation n'a pas reçue l'attention qu'elle mérite en tant que méthode pour apprendre la musique. Merriam avait sans doute tort de n'y voir qu' « un premier pas", pour affirmer ensuite que "des capacités spéciales requièrent un entraînement spécial», ou que "pour devenir un vrai musicien, il faut avoir suivi un apprentissage formel» (1964: 147, 150). A Hérat, c'est bel et bien l'imitation qui semble avoir constitué le noyau du processus d'apprentissage. Quand l'enfant imite, il apprend en reproduisant ce qu'il a observé. L'acquisition d'un savoir-faire n'était pas démarquée sous forme d'un processus d'instruction systématisé. L'information visuelle a ici une importance cruciale; souvent, il faut d'abord voir pour comprendre ensuite la manière de faire. L'imitation encourage l'enfant à se débrouiller tout seul : on ne lui explique rien. Les compétences variables distinguant les enfants des familles ordinaires de ceux des familles de musiciens sont imputables aux ressources musicales s'offrant à l'imitation, ainsi qu'à la manière dont cette dernière était encouragée.

Plusieurs facteurs contribuaient ainsi à enrichir l'environnement musical des enfants originaires des familles de musiciens professionnels. 1. Dès la plus tendre enfance, ils étaient fréquemment exposés à la musique. 2. Ils étaient 
plus motivés, parce que fortement encouragés à se livrer à des jeux de type musical et autorisés à se servir des instruments de musique. 3. Ils disposaient de modèles à imiter; ils avaient fréquemment l'occasion d'assister au jeu instrumental des adultes et de leurs frères et sœurs aînés, et d'observer attentivement leurs prouesses d'instrumentistes. 4. Ils avaient la possibilité de prendre une part active à la pratique musicale des adultes. 5. Ils grandissaient dans un environnement social centré sur la pratique musicale. Ces différences inhérentes à l'environnement musical suffisent pour expliquer le savoir musical variable qui différencie les enfants des deux catégories retenues, et il n'est donc nul besoin de rechercher des explications d'ordre génétique.

L'apprentissage par imitation est lourd de conséquences, puisqu'il signifie que l'enfant reproduit inévitablement ce qui se passe autour de lui. Voilà l'exemple d'une culture agissant en tant qu'"organisation en vue de transmettre des comportements fort complexes et spécialisés» (Gillin in Merriam 1964 : 162). Ce principe apparaît aussi dans le second contraste révélé par nos données, qui est celui entre filles et garçons. A Hérat, les relations entre les sexes jouaient clairement dans le domaine musical, si l'on songe à l'accès limité qu'avaient les femmes aux biens matériels et aux informations. Par comparaison avec les hommes, elles disposaient d'un éventail plus restreint d'instruments de musique: en effet, la plupart des femmes n'avaient accès qu'au dāireh. Leur connaissance de la théorie musicale formelle était pareillement limitée. Elles n'étaient en outre pas confrontées aux problèmes d'accordage parce qu'elles ne jouaient pas des cordophones qui exigeaient l'accordage minutieux des cordes sympathiques, et qu'elles n'accordaient pas leurs tabla. Leur musique était structurellement, c'est-à-dire du point de vue de la mélodie et du rythme, plus simple que celle des hommes. D'une manière générale, elle ressemblait davantage à une méthode de récitation de la poésie, centrée qu'elle était sur les paroles plutôt que sur leur "mise en musique». Enfin, les femmes n'avaient pas accès aux genres de la musique savante.

La musique domestique des femmes telle que nous l'avons décrite ici, représentait le style le plus simple de tous les styles de musique pratiqués par les adultes, tout en étant le plus largement répandu. Parmi les musiciens amateurs de Hérat, le nombre d'interprètes femmes excédait de loin celui des hommes. C'est pourquoi nous considérons cette musique comme l'expérience imprégnatrice majeure des enfants de Hérat, comme le principal réservoir de toute musique. Il est significatif à cet égard qu'elle était aussi celle des enfants, établissant ainsi une relation rendue durable par l'interaction constante entre les enfants et les femmes plus âgées de leur groupe de parenté. De plus, c'était le type de musique sanctionné par la religion et de ce fait admissible pour les enfants. De cette manière, la vie musicale des enfants, qui commençaient leur apprentissage par imitation en bas âge pour se préparer aux rôles respectifs qu'ils étaient appelés à jouer à l'âge adulte, préfigurait les univers distinctifs dans lesquels se mouvaient femmes et hommes, tout comme la différence entre la musique des professionnels et celle pratiquée en amateur. 


\section{Notes}

* Cette recherche a été financée par un subside de recherche post-doctoral du Social Science Research Council, attribué à John Baily lorsqu'il était research fellow au Département d'anthropologie sociale de la Queen's University à Belfast (1973-1978). Le présent essai a été rédigé pendant que John Baily était visiting research fellow à la School of African and Asian Studies de l'Université de Sussex (1986-1988); sa traduction française est due à Isabelle Schulte-Tenckhoff.

${ }^{1}$ Voir Sakata (1983, chap. 4) pour une discussion du concept afghan de la musique.

${ }^{2}$ Pour plus d'informations au sujet du terme de shauqi, voir Slobin (1976: 23-24).

${ }^{3}$ Un aperçu plus détaillé de la vie des musiciennes à Hérat figure dans Doubleday (1988).

${ }^{4}$ Pour plus de détails sur la vie des musiciens de Hérat, voir Baily (1988).

\section{Bibliographie}

\section{BAILY John}

1976 "Recent changes in the dutär of Herat». Asian Music 8(1) : 29-64.

1979 «Professional and amateur musicians in Afghanistan ». World of Music 21(2) : 46-64.

1988 Music of Afghanistan: Professional musicians in the city of Herat. Cambridge: Cambridge University Press (Cambridge Studies in Ethnomusicology) (with accompanying audio cassette).

DOUBLEDAY Veronica

1988 Three Women of Herat. London : Jonathan Cape.

MERRIAM Alan P.

1964 The Anthropology of Music. Evanston : Northwestern University Press.

SAKATA Hiromi Lorraine

1983 Music in the Mind: The Concept of Music and Musician in Afghanistan. Kent: Kent State University Press (with accompanying audio cassettes).

SLOBIN Mark

1976 Music in the Culture of Northern Afghanistan. Tucson: University of Arizona Press (Viking Fund Publications in Anthropology $N^{\circ} 54$ ). 